\title{
A muon-spin relaxation study of $\mathrm{BiMnO}_{3}$
}

\author{
T. Lancaster ${ }^{1}$, S.J. Blundell ${ }^{1}$, P.J. Baker ${ }^{1}$, F.L. Pratt ${ }^{2}$, W. \\ Hayes $^{1}$, I. Yamada ${ }^{3}$, M. Azuma ${ }^{4}$ and M. Takano ${ }^{4}$ \\ E-mail: t.lancaster1@physics.ox.ac.uk \\ ${ }^{1}$ Clarendon Laboratory, Oxford University Department of Physics, Parks Road, \\ Oxford, OX1 3PU, UK \\ ${ }^{2}$ ISIS Facility, Rutherford Appleton Laboratory, Chilton, Oxfordshire OX11 0QX, UK \\ ${ }^{3}$ Institut de Minéralogie et de Physique des Milieux Condensés Université Pierre et \\ Marie Curie, 75015 Paris, France \\ ${ }^{4}$ Institute for Chemical Research, Kyoto University, Uji, Kyoto 611-0011, Japan
}

\begin{abstract}
We present the results of muon-spin relaxation measurements on ferromagnetic $\mathrm{BiMnO}_{3}$. Below $T_{\mathrm{C}}=98.0(1) \mathrm{K}$ oscillations in the time-dependence of the muon polarization are observed, characteristic of a quasistatic magnetic field at a single muon site, allowing us to probe the critical behaviour associated with the magnetic phase transition. We are able to suggest candidate muon sites on the basis of dipole field calculations. Close to $T_{\mathrm{C}}$, fluctuations of the $\mathrm{Mn}^{3+}$ moments are characteristic of critical behaviour while there is a sharp crossover to a region of fast dynamic fluctuations at higher temperatures.
\end{abstract}

PACS numbers: 75.47.Lx, 75.50.Dd, 76.75.+i, 77.80.-e

Submitted to: J. Phys.: Condens. Matter

(25 October 2018) 


\section{Introduction}

The possibility of combining ferroelectric and magnetic order in a material is of great interest from the point of view of fundamental condensed matter physics and of technology [1]. Although the microscopic nature of materials usually excludes this coexistence, there are a growing number of systems where spontaneous electrical and magnetic polarization exist in the same phase. These are examples of multiferroic materials (the general term describing systems where more than one of the ferroic order parameters are nonzero in the same phase) and have been of growing interest in recent years [2, 3, 4]. The system $R \mathrm{MnO}_{3}$ (where $R$ is a trivalent ion) has been widely studied in this context due to the coexistence, in some of the members of this series, of ferroelectric and magnetic order. The nature of the $R^{3+}$ ion is central to determining the structural, ferroelectric and magnetic properties of members of this system.

$\mathrm{BiMnO}_{3}$ has been the subject of much research interest, partly because its relative structural simplicity makes it amenable to study using first-principals theoretical techniques [5]. Early experimental work on the material was sparse, in part due to the requirement of high pressures in the preparation of the compound, and reports of ferroelectricity were initially speculative [6]. A detailed structural study [7] suggested that the material has a highly distorted perovskite structure (monoclinic space group $C 2$ ), shown in figure (1), which is consistent with the existence of ferroelectricity. Subsequent theoretical [5] and experimental studies [8, 10, 11, 12] also pointed to a coexistence of ferromagnetic and ferroelectric ordering at low temperature. Ferroelectric ordering was proposed to take place below $T_{\mathrm{E}} \approx 770 \mathrm{~K}[11$ induced by a centrosymmetric to non-centrosymmetric structural transition, although it was also suggested [8] that the ferroelectricity accompanied a structural transition occurring at $450 \mathrm{~K}$ without a change in symmetry.

The different structure and magnetic properties of $\mathrm{BiMnO}_{3}$ in comparison to other $R \mathrm{MnO}_{3}$ manganites, (which are antiferromagnetic and either orthorhombic or hexagonal) has been ascribed to the presence of the lone pair on bismuth [9, 10] and the covalent nature of the $\mathrm{Bi}-\mathrm{O}$ bonds which introduce additional orbital interactions (in contrast to the other $R \mathrm{MnO}_{3}$ manganites, where the bonding is essentially ionic [5]). It was proposed that these interactions lead to orbital order below the centrosymmetric to non-centrosymmetric transition at $770 \mathrm{~K}$ [13]. The orbital order, in turn, fosters three dimensional ferromagnetic interactions in this material [10] causing the material

to undergo a ferromagnetic ordering transition at a Curie temperature $T_{\mathrm{C}}$, reported to be $T_{\mathrm{C}}=98-105 \mathrm{~K}[14,15,8,16]$. The magnetic ordering transition results in a collinear magnetic structure [10] where the $\mathrm{Mn}^{3+}$ ions $\left(3 \mathrm{~d}^{4}, S=2\right)$ align along the $b$ axis (figure (11)).

Recently, detailed stuctural studies have suggested that, in contrast to the state of affairs described above, $\mathrm{BiMnO}_{3}$ actually crystallizes in the centrosymmetric space group $C 2 / c$ at $300 \mathrm{~K}$ [16, 17, 18]. This is incompatible with the existence of ferroelectricity and may necessitate a major revision of our view of this material. For example, it 


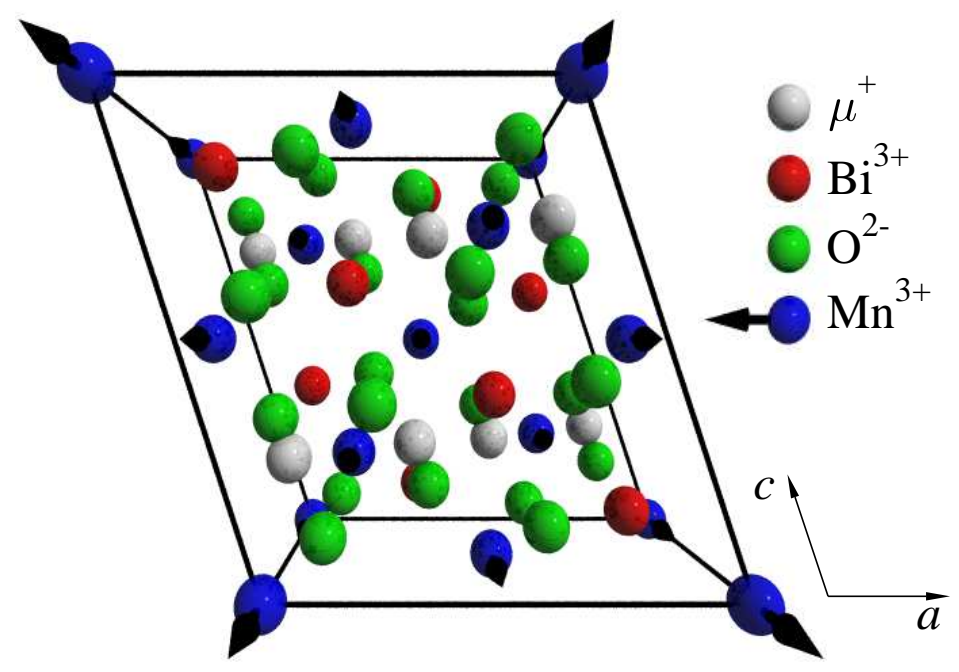

Figure 1. Structure of $\mathrm{BiMnO}_{3}(C 2$ space group) viewed down the $b$-axis. $\mathrm{Bi}$ is shown in red, $\mathrm{Mn}$ in blue, $\mathrm{O}$ in green and the proposed muon stopping sites in white (see main text). Arrows show the ferromagnetic spin structure found below $T_{\mathrm{C}}$, with the moments all along the b-axis.

has been suggested that the large dielectric anomaly observed near $T_{\mathrm{C}}$ may be linked to structural distortions occurring near this temperature regime rather than to effects related to broken inversion symmetry [18].

He we confine our discussion to the magnetic properties of $\mathrm{BiMnO}_{3}$. Muons are a sensitive probe of magnetism in condensed matter [19] and allow us to investigate magnetism from a local viewpoint. In this paper we present the results of a zero-field (ZF) muon-spin relaxation $\left(\mu^{+} \mathrm{SR}\right)$ study of the local magnetic properties of $\mathrm{BiMnO}_{3}$. We have probed the magnetic field distribution both below and above $T_{\mathrm{C}}$, allowing us to follow the temperature evolution of the order parameter in the magnetically ordered phase and to investigate the magnetic fluctuations in the paramagnetic phase. Our measurements also allow us to identify candidate muon stopping sites in the material.

\section{Experimental details}

A polycrystalline sample of $\mathrm{BiMnO}_{3}$ was prepared using the method reported previously [11], which involves heating a mixed powder of $\mathrm{Bi}_{2} \mathrm{O}_{3}, \mathrm{Mn}_{2} \mathrm{O}_{3}$ and $\mathrm{MnO}_{2}$ to $700{ }^{\circ} \mathrm{C}$ under $3 \mathrm{GPa}$ of pressure for 30 minutes. The structural character of the sample was checked using x-ray diffraction, while the bulk magnetic properties of the sample were measured using a commercial magnetometer.

In a $\mu^{+} \mathrm{SR}$ experiment, spin-polarized positive muons are stopped in a target sample, where the muon usually occupies an interstitial position in the crystal. The observed property in the experiment is the time evolution of the muon spin polarization, the behavior of which depends on the local magnetic field $B$ at the muon site, and which is proportional to the positron asymmetry function $A(t)$ [19]. ZF $\mu^{+} \mathrm{SR}$ measurements 

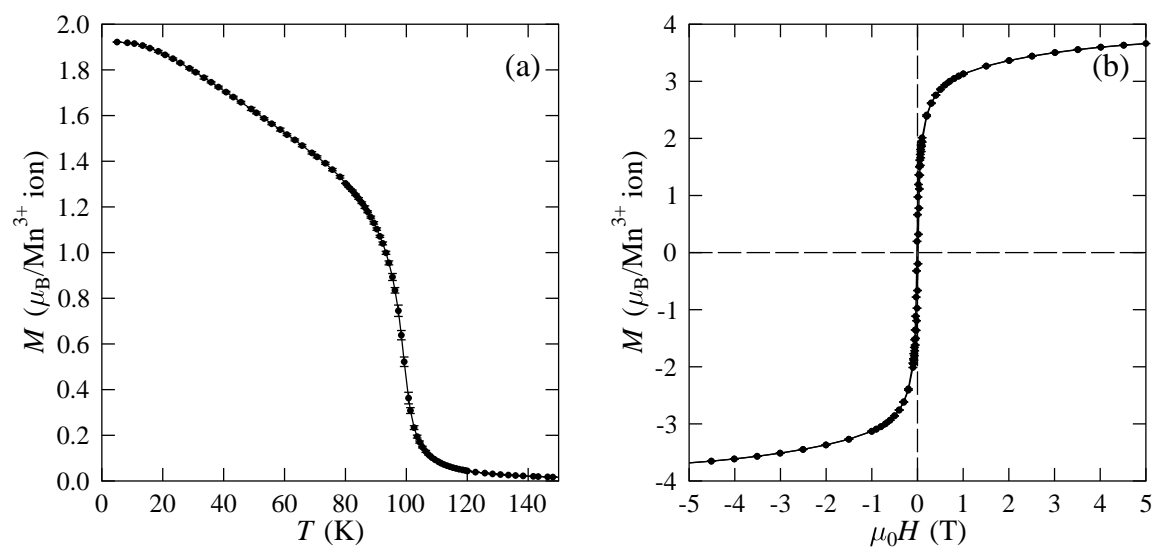

Figure 2. Magnetization of $\mathrm{BiMnO}_{3}$ : (a) measured as a function of temperature in a field of $100 \mathrm{mT}$; (b) measured as a function of applied field at $T=5 \mathrm{~K}$.

were made on $\mathrm{BiMnO}_{3}$ using the GPS instrument at the Swiss Muon Source $(\mathrm{S} \mu \mathrm{S})$, Paul Scherrer Institute, Villigen, Switzerland. The sample was wrapped in a silver foil packet (foil thickness $25 \mu \mathrm{m}$ ) and mounted on a silver backing plate. Silver is used as it possesses only a small nuclear moment and so minimizes any background depolarizing signal.

\section{Results}

Figure 2 shows the results of magnetization measurements made on our sample as a function of temperature and applied magnetic field. As seen in previous studies [14, 12, 8, 11, 16, the magnetic transition is evident close to $100 \mathrm{~K}$ (figure 2(a)). In an applied magnetic field the magnetic moment is seen, at low temperatures, to approach a saturation value of around $\sim 3.8 \mu_{\mathrm{B}}$ (figure 2(b)), as was also observed previously (see above).

Example ZF $\mu^{+} \mathrm{SR}$ spectra measured at $\mathrm{S} \mu \mathrm{S}$ on $\mathrm{BiMnO}_{3}$ are shown in figure 3 , At temperatures below $T=100 \mathrm{~K}$ and for times $t<0.2 \mu \mathrm{s}$, we observe oscillations in the asymmetry spectra (figure 3(a)). These are characteristic of a quasistatic local magnetic field at the muon site, which causes a coherent precession of the spins of those muons with a component of their spin polarization perpendicular to this local field; their presence provides strong evidence for the existence of long range magnetic order in this phase, in agreement with previous magnetic measurements. The frequency of the oscillations is given by $\nu_{i}=\gamma_{\mu} B_{i} / 2 \pi$, where $\gamma_{\mu}$ is the muon gyromagnetic ratio $\left(\equiv 2 \pi \times 135.5 \mathrm{MHz} \mathrm{T}^{-1}\right)$ and $B_{i}$ is the local field at the $i$ th muon site. In the presence of a distribution of local magnetic fields the oscillations are expected to relax with a depolarization rate $\lambda$. One precession frequency is observed in the measured spectra (see inset figure 3(a)), corresponding to a magnetically unique muon site in the material.

The spectra in this temperature regime were found to be best fitted, for times 

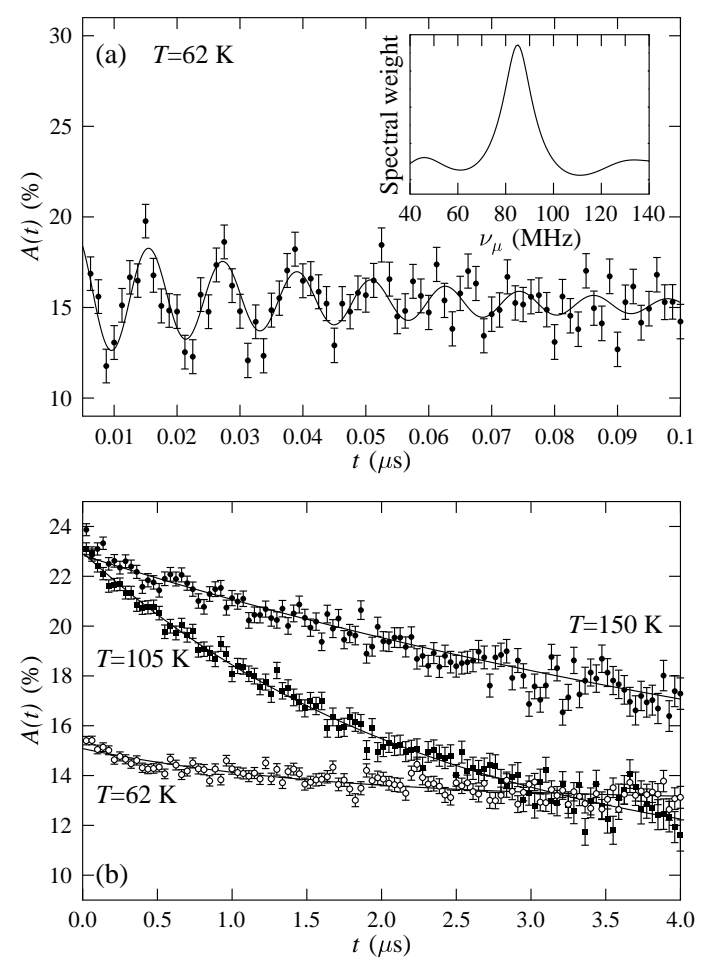

Figure 3. ZF $\mu^{+} \mathrm{SR}$ spectra measured for $\mathrm{BiMnO}_{3}$, showing fits as described in the text. (a) Example spectrum for times $0 \leq t \leq 0.1 \mu \mathrm{s}$ measured below $T_{\mathrm{C}}=98.0 \mathrm{~K}$. Oscillations are observed which are characteristic of quasistatic magnetic order at the muon site. Inset: Maximum entropy spectrum showing a single muon-spin precession frequency. (b) Spectra for times $0 \leq t \leq 4 \mu \mathrm{s}$. Above $T_{\mathrm{C}}$ the spectra are well described by a single exponential relaxation function. Below $T_{\mathrm{C}}$ only the longitudinal spin component is resolvable, with relaxation due to dynamic fluctuations.

$t<1 \mu \mathrm{s}$, to a function of the form

$$
A(t)=A_{\mathrm{bg}}+A_{\text {rel }}\left(\frac{1}{3} \exp \left(-\lambda_{1} t\right)+\frac{2}{3} \exp \left(-\lambda_{2} t\right) \cos \left(2 \pi \nu_{\mu} t+\phi\right)\right),
$$

where $A_{\text {rel }}$ represents the signal from the sample and $A_{\mathrm{bg}}$ represents a constant background contribution from those muons that stop in the sample holder or cryostat tail. The term multiplied by $1 / 3$ accounts for those components of the muon spin that are expected to lie parallel to the local magnetic field at the muon site in a polycrystalline material (and would therefore not be expected to give rise to an oscillatory signal). The transverse relaxation rate $\lambda_{2}$ was found to be constant for each temperature $T<T_{\mathrm{C}}$ and was fixed at a value $\lambda_{2}=7.0 \mathrm{MHz}$. A phase offset of $\phi=-115^{\circ}$ was also required to fit the oscillations, probably arising from the difficulty in exactly identifying $t=0$ in this measurement of a very fast precession signal. We note that the model that gives rise to equation (11) describes a material that is uniformly magnetically ordered throughout its bulk. In our $\mu^{+} \mathrm{SR}$ measurements we are unable to resolve any signal from a spin-glass-like phase that has recently been proposed to exist below $T_{\mathrm{C}}$ [16]. 
The temperature evolution of the frequency, extracted from fits to equation (1) to the measured data, is shown in figure 4(a). Fits to the phenomenological form $\nu_{\mu}=\nu_{\mu}(0)\left(1-\left(T / T_{\mathrm{C}}\right)^{\alpha}\right)^{\beta}$ yield $T_{\mathrm{C}}=98.0(1) \mathrm{K}, \nu_{\mu}(0)=117(1) \mathrm{MHz}, \alpha=1.1(1)$ and $\beta=0.35(1)$. We note that our value of $\beta$ is consistent with the 3D Heisenberg model. The parameter $\alpha$ is smaller than would be expected for ferromagnetic magnons (for which we expect $\alpha=3 / 2$ ). These parameters are robust when the fitting range is varied and attempts to fit only the low temperature portion of the data similarly fail to reveal the expected low temperature dependence. Although this may be attributed to the low concentration of data points in the limit $T \rightarrow 0$, we note that the form for the magnetic contribution to the heat capacity $\left(C_{\mathrm{m}} \propto T^{3 / 2}\right)$ expected for ferromagnetic magnons was not observed [16], which might suggest that the system is not amenable to a simple spin wave analysis.

Our estimate of $\nu_{i}(0)$ allows us to attempt to determine the muon sites in $\mathrm{BiMnO}_{3}$. The local magnetic field at a muon site $\mathbf{B}_{\mu}\left(\mathbf{r}_{\mu}\right)$ is usually dominated by the sum of dipolar contributions. Assuming the local field is due entirely to the dipole field of $\mathrm{Mn}^{3+}$ ions we may write

$$
\mathbf{B}_{\mu}\left(\mathbf{r}_{\mu}\right)=\sum_{i} \frac{3\left(\boldsymbol{\mu}_{i} \cdot \hat{\mathbf{n}}_{i}\right) \hat{\mathbf{n}}_{i}-\boldsymbol{\mu}_{i}}{\left|\mathbf{r}_{\mu}-\mathbf{r}_{i}\right|^{3}},
$$

where $\mathbf{r}_{\mu}$ is the position of the muon, $\boldsymbol{\mu}_{i}$ is the ordered magnetic moment of the $i^{\text {th }} \mathrm{Mn}$ ion and $\hat{\mathbf{n}}_{i}\left(=\left(\mathbf{r}_{\mu}-\mathbf{r}_{i}\right) /\left|\mathbf{r}_{\mu}-\mathbf{r}_{i}\right|\right)$ is the unit vector from the muon to the Mn ion at site $\mathbf{r}_{i}$. The positive muon's position is usually in the vicinity of the electronegative $\mathrm{O}^{2-}$ ions [20].

Dipole fields were calculated in a sphere containing $\approx 10^{5} \mathrm{Mn}$ ions with moments of $3.8 \mu_{\mathrm{B}}$ (that is, the value of the saturation magnetization per $\mathrm{Mn}^{3+}$ ion estimated from our magnetization measurements) aligned along the [010] direction [10]. A magnetic field consistent with $\nu_{i}(0)$ is found at several positions in the unit cell. Using the $C 2$ space group, candidate muon sites may be identified at the positions $(0.40,0.52,0.26)$ and $(0.10,0.67,0.24)$, placing muons either side of the $\mathrm{Mn}^{3+}$ ion located at $(0.2460 .563$ 0.243). Application of the symmetry elements of $C 2$ generates crystallographically equivalent sites at which the same local field is experienced. The result is that the magnetically equivalent candidate muon sites lie close to a $\mathrm{Mn}^{3+}$ ion near $z=0.25$ or $z=0.75$, as shown in figure 1, where 8 sites are evident per unit cell. Applying the elements of $C 2$ to either candidate site generates no positions at which a different local magnetic field is found, which is consistent with our observation that there is a unique local field at all muon sites in the ordered regime. The calculation was checked for several values of $\mathrm{Mn}^{3+}$ magnetic moment in the range $3.2 \mu_{\mathrm{B}} \leq \mu_{i} \leq 3.8 \mu_{\mathrm{B}}$ to account for the different values of the magnetic moment reported from previous work [8, 10, 11, 12, 16]. Very similar muon sites to those described above were found for all calculations, with the exact positions shifted by a maximum of $\sim 2 \%$ of the lattice parameters. Repeating this procedure using the space group $C 2 / c$ leads to little difference in the local magnetic field profile. In this case the muon position is given by $(0.60,0.71,0.50)$. Applying the symmetry elements of $C 2 / c$ gives similar muon sites to those described above, placing 

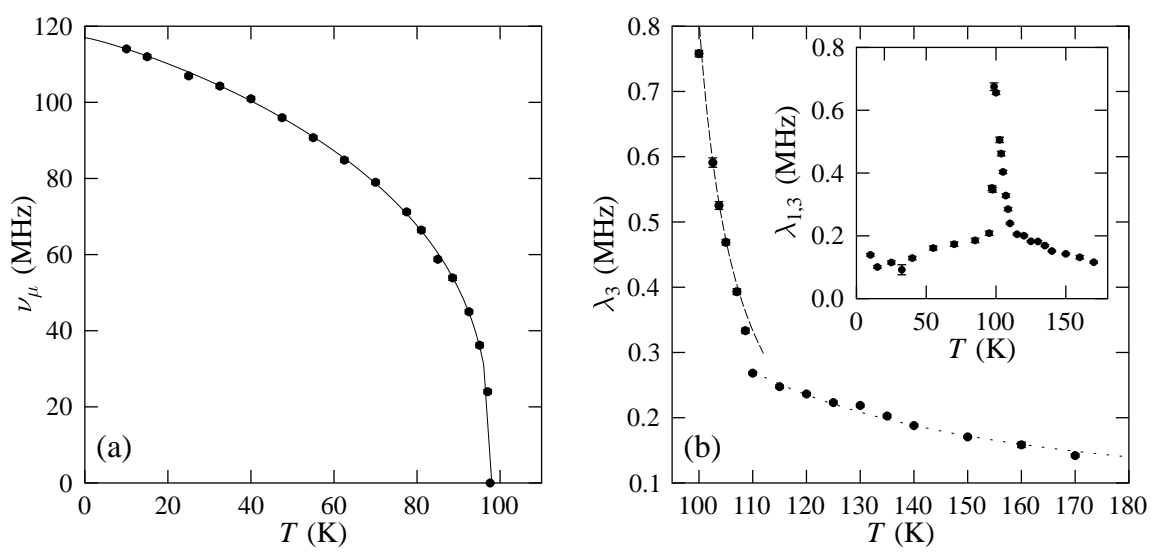

Figure 4. (a) Temperature evolution of the muon precession frequency below $T_{\mathrm{C}}=98.0 \mathrm{~K}$ with a fit to the phenomenological form $\nu_{\mu}=\nu_{\mu}(0)\left(1-\left(T / T_{\mathrm{C}}\right)^{\alpha}\right)^{\beta}$. (b) Variation of the relaxation rate $\lambda_{3}$ for spectra measured above $T_{\mathrm{C}}$. A large change in slope is seen close to $110 \mathrm{~K}$. A Fit is shown to a model of critical dynamics for $T<T_{\text {cr }}$ (dashed line; see text). The dotted line for $T>T_{\text {cr }}$ is a guide to the eye. Inset: Dynamic relaxation across the entire measured temperature range $\left(\lambda_{1}\right.$ for $T<T_{\mathrm{C}}$, $\lambda_{3}$ for $T>T_{\mathrm{C}}$ ) showing that the crossover is roughly symmetric about $T_{\mathrm{C}}$ (see main text).

muons on either side of the $\mathrm{Mn}$ ions $(0.75,0.75,0.5)$ and again leading to 8 magnetically equivalent sites per unit cell. It should be noted that in a ferromagnet such as $\mathrm{BiMnO}_{3}$ we would also expect contributions from the Lorentz field, demagnetization field and the hyperfine contact field which may mean that the situation is somewhat more complicated than considered here.

Above $T_{\mathrm{c}}$, the spectra are found to be purely relaxing (figure 3(b)) and are best described with a single exponential function

$$
A(t)=A_{\text {bg }}+A_{\text {rel }} \exp \left(-\lambda_{3} t\right),
$$

where $\lambda_{3}$ is a relaxation rate and $A_{\text {rel }}$ is constant. An exponential relaxation is usually indicative of dynamic fluctuations in the local magnetic field at the muon site [21]. In this case the relaxation rate may be written as $\lambda_{3} \propto(\Delta B)^{2} \tau$, where $\Delta B$ is the second moment of the local magnetic field distribution and $\tau$ is the correlation time. If the distribution of spin disorder within the system is temperature dependent we would expect a variation in $\Delta B$. More often, in the absence of magnetic or structural transitions, $\Delta B$ does not have a dramatic temperature variation and it is the fluctuation rate $\tau$ that varies with temperature. The variation of $\lambda_{3}$ with temperature is shown in figure 4(b) where we see a monotonic decrease in $\lambda_{3}$ with increasing temperature but with a significant change in slope around $T_{\mathrm{cr}} \approx 110 \mathrm{~K}$. This is indicative of a sharp crossover between regimes of slow dynamics (large $\tau$ ) below $T_{\text {cr }}$ and faster dynamics (small $\tau$ ) at higher temperatures. A clue to the origin of this behaviour may be found by comparing $\lambda_{3}$ to the relaxation at long times $(t<10 \mu \mathrm{s})$ for $T<T_{\mathrm{C}}$, corresponding 
to the relaxation rate $\lambda_{1}$ in equation (1), which also arises due to dynamic relaxation processes. This is facilitated by increasing the size of the time bins of the data so that the oscillations become unresolvable below $T_{\mathrm{C}}$ as shown in figure 3(b). The results of such an analysis are shown in the inset of figure 4 (b), where we see that $\lambda$ is roughly symmetrical in temperature about $T_{\mathrm{C}}$, with $\tau$ increasing dramatically for $\left|T-T_{\mathrm{C}}\right|<10 \mathrm{~K}$.

The sharp crossover seen in the dynamic relaxation rates $\lambda_{1}$ and $\lambda_{3}$ does not appear to correspond to features in the the bulk properties of $\mathrm{BiMnO}_{3}$. Given the symmetrical nature of the effect about the critical temperature, it seems unlikely that it is related to a structural transition (causing a change in the second moment of the local field distribution $\Delta$ ) of the sort that has been suggested to account for the dielectric anomaly near $T_{\mathrm{C}}$ [18]. Instead, it is probable that it is a different dynamic relaxation channel that determines the relaxation time $\tau$ on either side of the crossover. In a material where more than one relaxation channel coexists at a particular temperature, only the slowest (i.e. the channel described by the largest relaxation rate $\tau$ ) will dominate the muon-spin relaxation. This is expected to lead to relaxation with a single relaxation rate $\lambda$ as observed in this case. (In contrast, multiple relaxation rates only coexist in cases where the fluctuations are confined to spatially separate regions [22].) From our observation of a single relaxation rate, we may therefore infer that the fluctuations occur throughout the entire bulk of $\mathrm{BiMnO}_{3}$ at all temperatures. Furthermore, the fact that the amplitude $A_{\text {rel }}$ is constant across the measured temperature range shows that this crossover is intrinsic to the bulk of the material.

At temperatures above $T_{\text {cr }}$ dynamic fluctuations of the $\mathrm{Mn}^{3+}$ spins are probable, slowing as the temperature is decreased leading to an increase in $\lambda_{3}$. The nature of the dynamics causing the muon depolarization in this regime is not clear and its temperature dependence does not enjoy a form that may be described by a simple physical model. As the temperature is decreased below $T_{\mathrm{C}}$ another relaxation process, with a more dramatic temperature dependence, becomes the slowest channel and then dominates the measured relaxation rate $\lambda$. The symmetrical behaviour of $\lambda_{1,3}$ suggests that this process is caused by critical dynamics [23] in the region $\left(T_{\mathrm{C}}-\Delta\right) \leq T \leq\left(T_{\mathrm{C}}+\Delta\right)$, where $\Delta=T_{\mathrm{cr}}-T_{\mathrm{C}}$. Dynamic relaxation rates are proportional to $\sum_{\mathbf{k}} C(\mathbf{k}, \omega=0)$, where $C(\mathbf{k}, \omega=0)$ is the zero frequency correlation function for wavevector $\mathbf{k}$, describing the local fields at the muon site [23]. Calculations of $C(\mathbf{k}, \omega=0)$ lead us to expect $\lambda_{3}$ to diverge close to $T_{\mathrm{C}}$ according to $\lambda_{3} \propto\left|T-T_{\mathrm{C}}\right|^{-w}$, where $w$ may be related to the standard critical exponents [24] via $w=\nu(z+2-\eta-d)$ [23]. For a $d=3$ Heisenberg model we find $w=1.026$ [25], leading to the fit shown in figure 4 (b) for $T_{\mathrm{C}} \leq T \leq T_{\mathrm{cr}}$. This approach describes the data adequately, becoming worse, as should be expected, as we move away from $T_{\mathrm{C}}$. Finally, as the temperature is lowered below $T_{\mathrm{C}}-\Delta$ in the ordered regime, residual spin fluctuations become the slowest channel and therefore dominate $\lambda_{1}$.

These results suggest that the spin fluctuations in $\mathrm{BiMnO}_{3}$ are not trivial. Inelastic neutron scattering measurements may throw further light on the magnetic excitations in this system. 


\section{Conclusions}

We have carried out a $\mu^{+} \mathrm{SR}$ investigation of the multiferroic material $\mathrm{BiMnO}_{3}$. Our measurements show that the ferromagnetic transition occurs at $T_{\mathrm{C}}=98.0(1) \mathrm{K}$ with two regimes of dynamics separated by a sharp crossover at $T_{\mathrm{cr}} \approx 110 \mathrm{~K}$. We have also identified candidate muon sites in the material on the strength of dipole field calculations. Muons have been shown to be a useful and effective probe of magnetism in this system. The possibility of carrying out future muon studies in the presence of applied electric fields (for an example see [26]) presents opportunities for investigations of the microscopic mechanisms responsible for multiferroic behaviour in this class of materials.

\section{Acknowledgments}

Part of this work was carried out at the Swiss Muon Source, Paul Scherrer Institute, Villigen, Switzerland. We thank Alex Amato for technical assistance. This work is supported by the EPSRC. T.L. acknowledges support from the Royal Commission for the Exhibition of 1851.

\section{References}

[1] Hill N A and Fiebig M 2005 Science 309391

[2] Cheong S -W and Mostovoy M 2007 Nature Mater. 613

[3] Khomskii D I 2006 J. Magn. Magn. Mater. 3061

[4] Eerenstein W, Mathur N D and Scott J F 2006 Nature 442759

[5] Hill N A and Rabe K M 1999 Phys. Rev. B 598759

[6] Bokov V A, Grigoryan N A, Bryzhina M F and Kazaryan V S 1969 Bull. Acad. Sci. USSR, Phys. Ser. 33182

[7] Atou T, Chiba H, Ohoyama K, Yamaguchi Y and Syono Y 1999 J. Solid. State. Chem 145639

[8] Moreira dos Santos A, Parashar S, Raju A R, Zhao Y S Cheetham A K and Rao C N R 2002 Solid State Commun. 12249

[9] Seshadri R and Hill N A 2001 Chem. Mater. 132892

[10] Moreira dos Santos A, Cheetham A K, Atou T, Syono Y, Yamaguchi Y, Ohoyama K, Chiba H and Rao C N R 2002 Phys. Rev. B 66064425

[11] Kimura T, Kawamoto S, Yamada I, Azuma M, Takano M and Tokura Y 2003 Phys Rev B 67 180401(R)

[12] Chi Z H, Xiao C J, Feng S M, Li F Y, Jin C Q, Wang X H, Chen R Z and Li L T 2005 J. Appl. Phys. 98103519

[13] Yang C -H, Koo J, Song C, Koo T Y, Lee K -B and Jeong Y H 200673224112

[14] Sugawara F, Iida S, Syono Y and Akimoto S 1965 J. Phys. Soc. Japan 20 1529; Sugawara F, Iida S, Syono Y and Akimoto S 1968 J. Phys. Soc. Japan 251553

[15] Chiba H, Atou T and Syono Y 1997 J. Solid State Chem. 132139

[16] Belik A A and Takayama-Muromachi E 2006 Inorg. Chem. 4510224

[17] Belik A A, Iikubo S, Yokosawa T, Kodama K, Igawa N, Shamoto S, Azuma M, Takano M, Kimoto K, Matsui Y and Takayama-Muromachi E 2007 J. Am. Chem. Soc. 129971

[18] Montanari E, Calestani G, Righi L, Gilioli E, Bolzoni F, Knight K S and Radaelli P G (2007) Phys. Rev. B $\mathbf{7 5} 220101$ 
[19] Blundell S J 1999 Contemp. Phys. 40, 175

[20] Holzschuh E, Denison A B, Kündig W, Meier F P and Patterson B D 1983 Phys. Rev. B 275294

[21] Hayano R S, Uemura Y J, Imazato J, Nishida N, Yamazaki T and Kubo R, 1979 Phys. Rev. B 20 850

[22] Heffner R H, Sonier J E, MacLaughlin D E, Nieuwenhuys G J, Ehlers G, Mezei F, Cheong S -W, Gardner J S and Röder H 2000 Phys. Rev. Lett. 853285

[23] Hohenberg P C and Halperin B I 1977 Rev. Mod. Phys. 49435

[24] Binney J J, Dowrick N J, Fisher A J and Newman M E J 1992 The Theory of Critical Phenomena (Oxford: OUP)

[25] Pelisseto A and Vicari E 2002 Physics Reports 368549

[26] Storchak V G, Eshchenko D G, Lichti R L, and Brewer J H 2003 Phys. Rev. B 67121201 